\title{
Le basi filosofiche del Circolo Linguistico di Praga negli ANNi ‘60 DEL XX SECOLO ${ }^{1}$
}

\author{
Fabio RIPAMONTI \\ Università della Boemia meridionale, České Budějovice
}

\begin{abstract}
En): Within the Prague Linguistic Circle there is a small number of philosophical currents (structuralism, phenomenology, functionalism) that have influenced its activities from the classical period (1926-1938). The reflection on these currents was so profound that continued even in the 60's, proving fruitful comparisons with the Marxist dialectic and with the development of phenomenology in Czechoslovakia without betraying the original setting. In this way, the Circle was able to develop an original way of conceiving the language where it is possible to find influences from the thought of that period.
\end{abstract}

Keywords (En): Prague Linguistic Circle ; structuralism ; phenomenology ; functionalism ; dialectics

Parole chiave (It): Circolo Linguistico di Praga ; strutturalismo ; fenomenologia ; funzionalismo ; dialettica

\section{Introduzione}

Il Circolo Linguistico di Praga (CLP) rappresenta uno dei più significativi orientamenti europei nell'ambito dello strutturalismo e della concezione della lingua in senso funzionalistico che si siano espressi nell' arco del XX secolo. La sua genesi e il suo sviluppo è di particolare interesse per la storia della cultura poiché vanno di pari passo con i principali eventi che Praga ha vissuto durante l'ultimo secolo. La maggior parte dell'attenzione degli studiosi è stata attirata dal cosiddetto periodo classico (1926-1938), in cui il CLP seppe creare un'influente scuola soprattutto nel campo della fonologia, mentre le teorie sviluppate negli anni '60 del XX secolo sembrano trascurate dai lavori degli specialisti, nonostante anch'esse rappresentino un contributo di sicuro interesse per la linguistica.

Le teorie del CLP sviluppate nel periodo classico rimasero sostanzialmente immutate, almeno nei loro aspetti basilari, nelle epoche successive, segno evidente di come la riflessione dei suoi membri sia stata capace di sviluppare radici solide e profonde ma al contempo sufficientemente elastiche da permettere l'assorbimento delle novità che nel campo della linguistica erano andate man mano formandosi nel corso dei decenni successivi, senza per questo venir meno all'impostazione originale (VACHEK, 1999 : 82).

\section{Le radici del periodo classico}

Come ben noto, il CLP venne fondato nel 1926 grazie all'iniziativa di alcune personalità del mondo accademico praghese e di alcuni «stranieri», soprattutto russi, ma anche francesi e di lingua tedesca, i quali trovarono nella Praga della Prima repubblica, culturalmente dominata dal presidente-filosofo Tomáš Garrigue Masaryk, una città stimolante, galvanizzata in seguito alla rinnovata indipendenza

1 Le traduzioni delle citazioni da testi cechi sono dell'autore. 
politica, che offriva un ideale ambiente di lavoro, cosmopolita, aperto e pertanto sensibile e ricettivo alla circolazione delle più attuali idee del momento. Bisogna però ricordare che ancor prima della proclamazione della Cecoslovacchia a Praga si era già sviluppato un interesse verso gli studi linguistici, tanto da poter parlare persino di una «preistoria del CLP» (VACHEK, 1999 : 14), infatti Vilém Mathesius, fondatore e primo segretario del CLP, nel 1911 aveva già tenuto la sua celebre conferenza dal titolo O potenciálnosti jevů jazykových (MATHESIUS, 1982), che venne considerata da parte dei membri del CLP un importante punto di partenza ante litteram per i temi che successivamente sarebbero diventati centrali nei loro interessi e che già presentava notevoli punti di affinità verso ciò che Ferdinand de Saussure stava elaborando nello stesso periodo a Ginevra, senza che i due avessero avuto la possibilità non solo di conoscersi, ma nemmeno di essere informati sulle loro attività. Una volta pubblicato e diffuso il Cours de linguistique générale, giunto a Praga grazie a Sergej Karcevskij, che aveva assistito alle lezioni di Saussure a Ginevra, l'assimilazione delle teorie del linguista ginevrino fu pertanto un fatto quasi naturale, avvenuto per gradi e in modo differente a seconda dei membri, non senza un'interpretazione originale e critica dei diversi aspetti delle sue teorie. Il rapporto tra Saussure e il CLP è un tema che non ha trovato sempre d'accordo tutti gli studiosi, impegnati nel ricercare le eventuali influenze dell'uno sull'altro o il primato nell'elaborazione di alcuni concetti (primo fra tutti quello di «fonema») con risultati contrastanti ; quello che è però certo (ČERMÁK, 2003) è che si possono identificare sia elementi comuni al CLP e a Saussure (alcune distinzioni metodologiche del tipo sincronia-diacronia, langue-parole, paradigma-sintagma; il concetto di sistema e quello di «segno linguistico»), sia elementi tipici solo per Saussure (la nozione di valore e il concetto di linguaggio), oppure solo per il CLP (la nozione di funzione, il rapporto centro-periferia, la potenzialità del linguaggio e la prospettiva funzionale delle frase). Secondo VACHEK (1966 : 15-39), le principali remore da parte dei linguisti del periodo classico del CLP verso le teorie di Saussure riguardavano il concetto di parole, il quale a differenza di quello di langue non era stato sviluppato pienamente all'interno del Cours. Egli non fu capace, secondo Roman Jakobson, di conciliare il carattere sistematico della langue coi cambiamenti che avvengono in seno alla langue stessa e che si manifestano nella parole. Jakobson sottolineò la contraddizione e cercò di superarla con la nozione del «carattere terapeutico del cambio linguistico», secondo la quale ogni sistema linguistico in qualsiasi momento del suo sviluppo si trova per sua natura in un equilibrio instabile e si evolve continuamente mutando gli aspetti che avverte, per così dire, «malati». A parte queste precisazioni, si può affermare che tra gli anni '20 e ' 30 del XX secolo lo strutturalismo mise radici profonde a Praga, se si considera che a questa metodologia si continuò a fare riferimento anche nelle epoche successive, come appunto negli anni '60, durante i quali ci si dovette confrontare con le teorie marxiste allora ovviamente imperanti. Una definizione azzeccata per descrivere quegli anni si può trovare in Květoslav Chvatík (riportato in RAYNAUD, 1990 : 92) secondo il quale : «Si tratta di un concetto [quello strutturale presente nelle opere di autori cechi e slovacchi] che si può brevemente caratterizzare come strutturologia dialettica e storica. La struttura viene qui concepita come un processo dinamico, non contraddittorio e la comunicazione umana come un 
processo sociale di semiosi che non si può separare dal suo autore, l'uomo attivo e la sua prassi storica».

Una metodologia basata su opposizioni dialettiche all'interno del sistema linguistico è un procedimento tipico già per i fondatori del CLP (VACHEK, 1966 : 32-33), i quali l'avevano applicata con risultati significativi soprattutto nel campo della fonologia già a partire da Karcevskij, secondo il quale il valore di un segno linguistico acquista significato esclusivamente all'interno di un equilibrio instabile tra opposizioni su vari livelli (significato/significante, generale/individuale, astratto/concreto, virtuale/situazionale, antico/moderno e così via). Questa stessa metodologia non era estranea nemmeno a SAUSSURE, come quando ad esempio afferma che «nella lingua non vi sono se non differenze» (2007 : 145) oppure che il valore di un elemento linguistico non esiste in sé ma in rapporto con gli altri elementi del sistema (op. cit. : 136-148). Pertanto il tema principale per lo strutturalismo è capire che tipo di sistema sia il linguaggio, probabilmente il più complicato di tutti, il «sistema dei sistemi» (VACHEK, 1999 : 27-30).

\section{1 Gli apporti della fenomenologia alle teorie del CLP}

Insieme allo strutturalismo, la fenomenologia di Edmund Husserl costituì un altro riferimento importante per buona parte dei membri fondatori del CLP, in particolar modo per via del carattere programmatico di questa corrente, orientata a dare un fondamento solido alla conoscenza, e diffusasi nel momento in cui la linguistica voleva fornirsi di un paradigma stabile. Lo stesso destino personale di Husserl sembra legato a doppio filo alle sorti del mondo cecoslovacco : nacque infatti a Prostějov in Moravia, conobbe Masaryk, il quale lo orientò agli studi di filosofia, mentre era studente a Vienna, ebbe un proficuo rapporto con Jan Patočka, considerato il suo ultimo discepolo, e nel novembre 1935 , nei tempi in cui meditava di ritornare nella sua vecchia madrepatria a causa del clima ostile che si stava formando in Germania, tenne un ciclo di conferenze a Praga durante le quali ebbe anche l'occasione di intervenire all'interno del CLP sul rapporto della filosofia con le scienze dello spirito e con la lingua (BLECHA, 2003 : 46). L'interesse verso Husserl e la fenomenologia in generale era molto vivo soprattutto nella frangia russa del CLP ancor prima dell'arrivo a Praga, in particolar modo in Jakobson ; a quanto pare, ciò che aveva attirato il suo interesse era il carattere antipsicologistico delle teorie fenomenologiche, che permise alla linguistica di sviluppare un processo orientato altresì all'affrancamento dalla concezione positivistica incarnata dal metodo neogrammatico allora ancora piuttosto vivo. Oltre a questo principio, la fenomenologia legittimava il ricorso all'uso della coscienza come determinante nell'atto di fondare la linguistica come scienza e si affiancava alla concezione strutturale della lingua così come si stava sviluppando in seguito all'assimilazione del Cours grazie alla considerazione del linguaggio come un sistema strutturato, formato da un tutto con le sue parti, obbedienti a leggi a priori in cui la teoria dell'intenzionalità fungeva da base per il procedimento funzionalista (BARKOVA, 2008 : 60). Fenomenologia e strutturalismo, dunque, sono entrambi impegnati nell' analisi della logica interna del sistema, del nucleo strutturale atto a spiegare il funzionamento della lingua (RAYNAUD, 1990 : 70). BARKOVA (2008 : 58-59) si spinge ancora più avanti affermando che il concetto di «struttura» nel CLP è concepito come un vero e 
proprio sistema fenomenologico che estrae il suo oggetto di indagine dal mondo reale, il quale non si presenta sotto forma di caos disorganizzato ma come una formazione ordinata ; i fenomeni linguistici precedono l'investigazione, pertanto è necessario indagare le loro relazioni interne e determinare le leggi sistematiche che ne emergono. La differenza tra strutturalismo in senso classico e strutturalismo praghese, forse esageratamente definito da questa autrice come «fenomenologico», riguarda i compiti della scienza linguistica : secondo Saussure il sistema del linguaggio deve essere costruito, per i praghesi, invece, è un oggetto ontologicamente strutturato che, formando un tutto, attende solo di essere scoperto.

\section{2 La concezione funzionalista della lingua}

Ciò che caratterizza maggiormente la peculiarità dello strutturalismo praghese rispetto ad altri è la sua interpretazione in chiave funzionalistica. Il concetto di funzionalismo era già ben chiaro e presente nelle tesi del CLP pubblicate nel 1929, ma ciò nonostante sembra difficoltoso risalire chiaramente a una sua genesi (DANEŠ, 1987 : 3-5 ; NOVÁK, 1968 : 291). È tuttavia naturale ritenere che il funzionalismo del CLP fosse ben ancorato al pensiero europeo dei primi anni del $\mathrm{XX}$ secolo se lo si mette in relazione a concetti quali ad esempio quelli di «compito, fine, obiettivo» (NOVÁK, 1968 : 292) che circolavano in quel periodo anche in altre discipline. L'humus praghese era insomma già orientato verso questa metodologia (ŠOLTYS, 1991), che seppe dare risultati significativi soprattutto nell'architettura e nelle arti plastiche in genere, ma anche nella riflessione sull'economia, come anche dimostrato da un'opera di Karel ENGLIŠ (1930), uno dei più importanti economisti cechi : questo libro, che a volte viene indicato come un riferimento specifico per il funzionalismo del CLP (HAJIČOVÁ, 2003 : 28), fu in grado di provocare parecchie discussioni all'epoca, alle quali probabilmente aveva partecipato anche Mathesius (DANEŠ, 1987 : 5-7). Oltre a questi aspetti, va pure sottolineato che l'utilizzo del concetto di funzionalismo fattone dai vari autori del CLP non era sempre univoco e ha dato adito a interpretazioni a volte discordanti. Secondo DANEŠ (1987 : 9) ci sono almeno cinque varianti in base alle quali il concetto di funzionalismo si è sviluppato all'interno del CLP :

a) Il requisito metodologico per cui bisogna partire dai bisogni della comunicazione e dell'espressione per analizzare con quali mezzi questi bisogni sono soddisfatti. Già in MATHESIUS (1929) si accenna alla necessità di considerare il punto di vista del parlante e i suoi bisogni comunicativi come base dei principi del funzionalismo, tuttavia NovÁK e SGALL (1968 : 295) mostrano i problemi di una simile analisi : difficoltoso è valutare ad esempio se questi bisogni siano o no universali, se siano interni o esterni alla lingua oppure individuare quali siano le loro unità minime.

b) Le funzioni esterne della lingua, elo degli enunciati. Questo è un punto ben presente nel CLP a partire dalla nota tripartizione di Bühler tra Soggetto (Ausdruck) / Destinatario (Appel) / Rappresentazione (Darstellung), poi sviluppatosi, grazie a una serie di aggiustamenti e di precisazioni da parte di altri autori non solo praghesi, nel famoso schema di JAKOBSON costituito da sei 
funzioni (1966 : 185 e segg.), che ricopre sinteticamente tutte le varianti teoricamente possibili della comunicazione umana.

c) Le funzioni delle unità del sistema linguistico. Negli scritti dei praghesi non si trovano indicazioni precise su quale livello del sistema linguistico il concetto di «funzione» sia orientato, benché già nelle tesi del '29 si dica che la lingua è un sistema orientato a un fine. Nel periodo classico questo principio veniva applicato soprattutto nel campo della fonologia, ma successivamente è stata sottolineata l'esistenza di differenze tra la fonologia della parola e la fonologia della frase, dato che l'intonazione e la fonetica possono cambiare il senso di un enunciato. Nell'ambito delle unità di segno, la funzione tocca tutti i livelli del sistema. Queste funzioni possono essere semantiche, o secondo un'altra terminologia pragmatiche, oppure grammaticali, morfologiche, sintattiche o costruzionali (ad es. i connettori).

d) La prospettiva funzionale della frase. Anche questo livello di analisi fu introdotto compiutamente da MATHESIUS (1939), il quale per primo parlò della necessità di distinguere il significato grammaticale da quello funzionale nell'interpretare un enunciato. Successivamente si sono moltiplicati i contributi in questa direzione, orientati a precisare ancora meglio questo livello di analisi, tanto da diventare uno dei filoni più produttivi all'interno del CLP, studiato a partire dagli anni '60 del XX secolo soprattutto da Jan Firbas e da altri ancora, anche in prospettiva generativista (HAJIČOVÁ, 2003 : 82-113).

e) La spiegazione funzionale dello sviluppo linguistico. In diacronia, il concetto di funzione risulta utile per spiegare in che modo un sistema linguistico tende a ristabilire il suo equilibrio durante l'evoluzione, principio complementare all'idea del «cambiamento terapeutico» di Jakobson.

\section{3 L'integrazione tra funzionalismo e dialettica}

Daneš afferma la necessità di integrare il funzionalismo praghese con la dialettica di Hegel e Marx secondo le modalità presentate in un articolo di Pierre VAN DER BERGHE (1963), dove si vuole dimostrare che le due correnti, nonostante le specifiche limitazioni, sono tra di loro complementari e potrebbero fornire un modello completo sullo sviluppo della società. Il modello che fa riferimento al funzionalismo strutturalista si basa sui seguenti concetti : 1) le società devono essere analizzate in modo olistico 2) le cause sono molteplici e reciproche 3) anche se l'integrazione non è mai perfetta, ogni società si trova in un equilibrio instabile che tende verso la stabilità e l'inerzia 4) l'equilibrio, anche se irraggiungibile, rimane il fine ultimo di ogni sistema 5) il cambio avviene progressivamente, quando ci sono rivoluzioni solitamente sono superficiali e non intaccano la sostanza del sistema 6) il cambio avviene solitamente per tre motivi : aggiustamento del sistema a un cambio esogeno, crescita attraverso una differenziazione strutturale e funzionale, innovazioni da parte dei membri del gruppo 7) il consenso è un fattore importante (op. cit. : 696). Il modello funzionalista basato sull'equilibrio non può essere soddisfacente per analizzare i fenomeni sociali poiché la reazione a uno stimolo esterno non è sempre di aggiustamento, dato che un sistema può mantenere per molto tempo un alto livello di cattiva integrazione. Il funzionalismo così inteso non è in grado di rispondere ad altre questioni, come ad esempio quali siano le forze che producono il cambio oppure se sia possibile prevederne la direzione. Al contrario, introducendo i 
principi della dialettica sulla base del funzionalismo, si può creare un modello più soddisfacente dello sviluppo della società. La dialettica di tipo hegeliano ha il vantaggio di ricordare che il cambio è inerente alla natura stessa delle idee, ma possiede anche il difetto di basarsi su un bipolarismo forzato che non corrisponde alla realtà. Tuttavia tra funzionalismo e dialettica esistono diversi punti di convergenza : entrambe queste correnti hanno un visione olistica della realtà ; sono orientate ad analizzare l'evoluzione sociale, che per il funzionalismo si basa sul principio del consenso mentre per la dialettica hegeliana-marxistica si basa sul principio del conflitto ; infine entrambi si ispirano a un modello di equilibrio, dove la classica tricotomia «tesi-antitesi-sintesi» non è altro che una formula adatta a rappresentare la ricerca dell' equilibrio stesso (op. cit. : 699-700).

\section{La Seconda Scuola di Praga}

Durante gli anni '60 del XX secolo gli orientamenti filosofici di cui si è parlato precedentemente (strutturalismo, fenomenologia, funzionalismo, dialettica) continuano ad avere un loro valore all'interno di ciò che può essere definita la «Seconda Scuola di Praga» (SORNICOLA, 1991 : 23). Successivamente al secondo conflitto mondiale e al conseguente mutamento del clima politico, il CLP si trovò a operare in condizioni particolarmente ostiche. Oltre alle difficoltà dovute a fattori esterni, si aggiunsero la morte di TRUBECKOJ (1939) e di MATHESIUS (1945), e l'emigrazione di Jakobson negli Stati Uniti. Dal punto di vista teorico, le difficoltà del CLP furono dovute alla diffusione nella linguistica russa del dopoguerra delle convinzioni di Nikolaj Jakovlevič Marr, le uniche considerate accettabili dall'ideologia ufficiale, che si ponevano contro tutte le concezioni linguistiche cosiddette «borghesi», quindi anche contro quella praghese. Per fortuna già nei primi anni '50 nella stessa Russia venne intrapresa una sistematica critica del marrismo, grazie alla quale venne stabilito ufficialmente che questa corrente aveva compromesso il buon nome della linguistica sovietica agli occhi di tutto il mondo. Tuttavia in questo nuovo clima non si arrivò alla naturale riabilitazione del CLP, ma nella pratica si cercò di liquidarlo sostituendolo con la «Associazione linguistica di boemistica, slavistica e di linguistica generale» (Jazykovědné sdružení pro problematiku bohemistickou, slavistickou i obecně lingvistickou) e con il «Circolo di filologia moderna» (Kruh moderních filologů), in cui però Bohumil Trnka riuscì a ricostituire il «Gruppo per la linguistica funzionalista»(Skupina pro funkční jazykozpyt), il quale a partire dal $1957 \mathrm{fu}$ capace di ottenere una significativa attenzione all'interno delle discussioni sullo strutturalismo che si svolgevano nell'Unione sovietica. Soprattutto per merito di Bohuslav Havránek, che si impegnò a calmare la confusione che regnava nella linguistica postmarristica, e grazie al nuovo clima politico instauratosi nel Paese durante gli anni '60 del XX secolo fu inaugurata una nuova era nella storia del CLP. Il primo passo fu la pubblicazione nel 1960 del «Dizionario linguistico della Scuola di Praga» (Lingvistický slovník Pražské školy) da parte di Vachek, tradotto immediatamente in russo, ma anche e soprattutto il rinnovamento, seppur sotto un titolo opportunisticamente un po' diverso dall'originale, dei Travaux linguistiques de Prague in quattro volumi («L'École de Prague d'aujourd'hui» - 1964 ; «Les problèmes du centre et de la périphérie du système de la langue» - 1966 : «Études 
structurales dédiées au VIe Congrès des slavistes» - 1968 ; «Études de la phonologie, typologie et de la linguistique générale» - 1971). La relativa libertà che si poteva godere nella Cecoslovacchia del periodo favorì una feconda serie di contatti e di collaborazioni con l'estero, soprattutto con gli Stati Uniti, dove VACHEK poté pubblicare le opere «Prague School Reader in Linguistics» (1964) e «The Linguistic School of Prague» (1966) ; inoltre ben presto fu possibile entrare in contatto con le novità nel campo, in particolar modo con la grammatica generativista di Noam Chomsky, verso la quale non mancarono né critiche né proficue interpretazioni delle sue teorie. In seguito all'occupazione della Cecoslovacchia da parte delle truppe del Patto di Varsavia nell'agosto del 1968, la situazione per il CLP tornò a essere di nuovo difficoltosa per non dire impossibile: i Travaux vennero soppressi e fu impedito a tutti i membri di partecipare collettivamente a qualsiasi iniziativa, sia in madrepatria che all'estero, sancendo di fatto la fine del CLP fino alla caduta del regime nel 1989 (VACHEK, 1999 : 64-74).

\subsection{I tentativi di conciliazione tra fenomenologia e teorie marxiste a supporto degli studi linguistici}

Nel clima politico degli anni '60 lo stesso destino del CLP toccò anche alla fenomenologia, che era entrata in crisi dopo il secondo conflitto mondiale in quanto filosofia «borghese» e di matrice tedesca, ma che nella nuova libertà di quegli anni seppe produrre risultati interessanti non solo nel confronto col marxismo ma anche con lo strutturalismo. Il rappresentante più significativo della fenomenologia del secondo dopoguerra, Jan Patočka, considerato il filosofo ceco più importante del $\mathrm{XX}$ secolo, non sembra aver avuto dei contatti diretti con il CLP, tuttavia non è da escludere che entrambi fossero informati sulle reciproche attività. Un interessante documento dell'interesse di Patočka verso il CLP si può scorgere nella recensione al volume di Elmar HOLENSTEIN «Roman Jakobsons phänomenologischer Strukturalismus» (1975), in cui viene effettuata un'analisi puntuale dei parallelismi tra fenomenologia, strutturalismo e dialettica. Patočka afferma che la fenomenologia e lo strutturalismo non solo non sono contrari ma si basano sugli stessi principi, addirittura in Jakobson è possibile osservare come la fenomenologia sappia sviluppare un'apertura verso altre discipline. Patočka considera l'inizio di Jakobson come filosofo non nell'opposizione ai neogrammatici, bensì nel superamento dell'opposizione saussureana di diacronia e sincronia, poiché così facendo è arrivato a realizzare un vero e proprio programma dialettico : («Unire in un concetto solido, ma allo stesso tempo elastico, di sistema l'idea di «opposizione» con quella relativa ai rapporti alla base del suo fondamento è un significativo atto di filosofia, per quanto questo venga alla luce sotto forma di strutture linguistiche» PATOČKA, $2009: 620)$. Nelle note a commento di questo passo, che successivamente verranno cancellate, afferma : «Da qui deriva la fenomenologia, simile a quella di Hegel. Senza opposizioni nella loro unità non è possibile nessun fatto linguistico, e questo a ogni livello del sistema. Da porsi la domanda se la fenomenologia di Husserl non sia un'introduzione all' analisi dialettica dei fatti linguistici» (op. cit. : 621, nota 479). A Jakobson viene recriminato di aver dato troppa importanza alla forma, al ruolo materiale dei fonemi e degli altri elementi del sistema linguistico, ma in realtà egli 
ha saputo andare oltre la tipica opposizione tra forma e contenuto, tema tradizionale dell'indagine linguistica, facendo derivare da ciò la vera sistematicità della lingua, il suo essere sistema, che viene creato dalla tensione di elementi in opposizione e non dalla sua considerazione come una sostanza unica.

\section{2 La conferenza di Liblice «I problemi della linguistica marxistica»}

Il momento decisivo per comprendere la rinnovata atmosfera degli studi linguistici in Cecoslovacchia dopo le difficoltà del periodo precedente va certamente indicato nella conferenza tenuta a Liblice nel 1960 dedicata ai problemi della linguistica marxistica (BĚLIČ, 1962) a cui parteciparono con interessanti contributi praticamente tutti coloro che da lì a qualche anno avrebbero dato di nuovo vita al CLP. Nel primo di questi contributi, Havránek discute in modo programmatico della metodologia che la linguistica marxista dovrebbe utilizzare ispirandosi al materialismo dialettico. Questo metodo è in grado di preservare la ricerca linguistica dal pericolo di ricadere in due estremi, individuati nel neopositivismo da una parte e nell'idealismo dall'altra, inaccettabili entrambi per il fatto che il primo privilegia esclusivamente ciò che è comune e il secondo invece ciò che è particolare, trascurando così la naturale condizione dialettica di ogni espressione del mondo materiale, compresa la lingua : («I rapporti dialettici esistono nei fenomeni sociali e studiandoli arriviamo a scoprirli. L'analisi empirica di questi fenomeni, se condotta veramente in modo scientifico, deve giungere a rivelare la loro corretta dialettica, la cui conoscenza dei principi generali è ovviamente di fondamentale ausilio in questo processo». HAVRÁNEK, 1962 : 19). Il sistema linguistico è un fenomeno sociale da analizzare non in modo meccanico, ma cercando di osservarvi in quale rapporto si posizionano le categorie dialettiche in opposizione, dato che ogni sistema di questo tipo vive da sempre una tensione e una contraddizione solamente apparente, consistente nel fatto che una lingua per essere viva deve cambiare, ma contemporaneamente deve essere stabile, altrimenti la comunicazione non potrebbe avvenire. Il metodo dialettico è in grado di superare questa apparente aporia e la linguistica marxista deve continuare su questa linea : («Un problema più complicato e dibattuto riguarda il rapporto tra la sistematicità della lingua e il suo funzionamento, il processo del discorso, il concreto 'fatto linguistico' che rappresenta allo stesso tempo un conflitto dialettico tra strumento e prodotto [...] È stato sottolineato qui il principio dell'unità tra forma e contenuto (significato) nel linguaggio e in questa unità non si può intravedere il rapporto tra ciò che è comune nel contenuto e ciò che è particolare nella forma, al contrario l'unità di forma e contenuto è sottostante al rapporto tra comune e particolare, tra astratto e concreto, sia nel sistema che nel funzionamento della lingua», op. cit. : 15-17). Questa tipologia di metodo non era una novità per i membri del CLP (KARCEVSKIJ, 1929), ma si potrebbe osservare che l'applicazione della dialettica permise loro di fornirsi di un quadro teorico uniforme.

\section{3 Gli sviluppi della fenomenologia nella Cecoslovacchia degli anni '60}

Il tentativo di trovare una conciliazione con le teorie marxistiche evidenziandone i tratti comuni non era una tendenza esclusiva della linguistica, ma era tipico anche per i futuri fenomenologi formatisi nei seminari organizzati da Patočka in forma privata a causa del suo allontanamento dal mondo accademico 
alla metà degli anni '60 (BLECHA, 2003 : 92). Tra i numerosi che vi parteciparono andrebbero ricordati soprattutto Antonín Mokrejš e Karel Kosík, i quali nonostante avessero avuto una formazione marxistica non disdegnavano il confronto con altri orientamenti filosofici. Mokrejš infatti pubblicò nel 1969 una monografia sulla fenomenologia dal titolo «Fenomenologie a problém intersubjektivity» [La fenomenologia e il problema dell'intersoggettività], dove tentò un confronto critico col marxismo. Questo autore sostiene che il marxismo non dovrebbe ignorare la fenomenologia, ma prenderla come una sfida utile per superare il dogmatismo, inoltre viene sottolineato in questa opera l'elemento dinamico presente in Husserl nel rapporto tra la realtà e l'io. Molto più complessa è invece la figura di Kosík, solitamente non ricordato come fenomenologo, anche se la sua tendenza ad analizzare l'uomo come essere economico si può collegare, ad esempio, alla filosofia di Martin Heidegger, già egli consapevole di alcuni parallelismi col marxismo. Anche Kosík aveva partecipato alla conferenza di Liblice già citata, nonostante il suo intervento non fosse strettamente collegato a temi linguistici ma vertesse sui concetti di sistema e di struttura (KosíK, 1962). Entrambi erano centrali nella riflessione degli anni '60 in Europa anche al di fuori del marxismo tout court e riecheggiavano nei lavori dei membri del CLP degli stessi anni, seppur in modo indiretto. Innanzitutto per Kosík bisogna considerare che ogni forma di conoscenza è caratterizzata dal suo essere un sistema aperto. Nelle epoche precedenti era opinione comune che la conoscenza fosse un percorso univoco, basato sulla conferma e sullo sviluppo dei principi del passato, ma ormai grazie allo sviluppo delle scienze e ad innovazioni come le geometrie non euclidee, la teoria della relatività e la fisica quantistica, la modalità della conoscenza non è più rappresentabile come una linea continua, bensì come un eterno movimento in avanti e indietro. La scienza non è un accumulo di fatti, deve presentare un carattere esplicativo, in questo senso si struttura come una dialettica tra fatti empirici, e quindi concreti, e principi, che servono a fornire una spiegazione dei primi. Quando si scoprono nuovi fatti che contraddicono le teorie vigenti allora bisogna cambiare queste ultime, in questo senso la scienza è un sistema aperto che deve rimanere tale per poter accettare ciò che accadrà nel futuro. Allo stesso tempo però deve anche conservare un carattere chiuso che tuttavia conservi un rapporto dialettico verso l'aspetto di apertura, altrimenti la scienza perderebbe il suo carattere esplicativo. Una concezione molto simile riecheggia in quella del linguaggio in quanto sistema così come elaborata all'interno del CLP : un sistema linguistico è per definizione in un equilibrio instabile, in perenne tensione tra tradizione e innovazione e tende a preservare questa condizione poiché gli garantisce di poter evolversi e adattarsi ai cambiamenti che incontra nel suo sviluppo (DANEŠ, 1966 : 12-13). Kosík continua nel suo articolo ricordando come lo sviluppo delle scienze nel XX secolo abbia portato alla conquista progressiva di nuovi campi di indagine per la filosofia e alla consapevolezza che in questo progresso si stia formando in modo sempre più solido la convinzione che campi lontani tra di loro funzionino secondo gli stessi principi. La consapevolezza che la realtà è dialettica ha fatto sì che si stia cercando di costruire una teoria generale valida in assoluto e che dia ragione del funzionamento di un sistema, compreso quello linguistico : «L'esistenza di similitudini strutturali nei più diversi settori di ricerca si fonda sul fatto che tutti i 
campi di analisi della realtà obiettiva si comportano come sistemi, ossia dei complessi di elementi che si influenzano reciprocamente» KosíK, 1962 : 29). Solo una comprensione dialettica dell'ontologia del sistema e della struttura permette di evitare gli estremi del formalismo matematico e della metafisica (op. cit. : 30-31). Più approfonditamente KOSÍK si sofferma sul concetto di «totalità», intesa come dialettica tra legge e caso, tra natura interna e esterna. Già Marx aveva ripreso questo concetto e ne aveva fatto il centro del materialismo dialettico : per Marx la «totalità del concreto» significava che ogni fenomeno sociale poteva essere spiegato e compreso solo in relazione al tutto, compito della filosofia era capire allora se l'idea di totalità non potesse implicare in sé quelle di struttura e di sistema in un senso dialettico. Il concetto di totalità è sempre stato importante negli autori d'ispirazione marxista per analizzare la società. In Cecoslovacchia iniziò ad attirare l'attenzione già negli anni '30 del XX secolo, nel periodo della polemica di Kurt KONRAD contro lo strutturalismo (1934), dove questo autore opponeva la concretezza della totalità marxista a quella astratta dello strutturalismo e separava il concetto di struttura da quello di strutturalismo, il quale così intenderebbe la struttura come un' applicazione idealistica, superficiale e unidirezionale. Il procedimento dialettico avrebbe allora il valore di ravvivare l'analisi strutturalistica in senso classico, quest'ultima colpevole, secondo Konrad, di non concepire la struttura in modo soggettivo osservando in essa la presenza dell'uomo in senso storico, ma di risolversi in un'analisi feticizzata al centro della quale vi sarebbe un astratto e inesistente essere umano che sostituirebbe l'individuo storicamente inteso. Su questa linea si muoveva anche il CLP, visto che non ha mai tralasciato di fare riferimento alla realtà concreta dei parlanti, peculiarità sottolineata in più occasioni sia in contrasto con la linguistica descrittivista di stampo americano che verso lo strutturalismo di Copenaghen rappresentato da Louis Hjelmslev (SKALIČKA, 1948).

KosÍK ritorna su questi temi anche nella sua opera probabilmente più importante, «Dialektika konkretního» (1963). In questo testo si afferma che alla dialettica interessa ricercare la sostanza, la «cosa in sé», che non si presenta mai però agli occhi degli uomini in modo diretto, bensì attraverso i fenomeni. Questi sono in grado di mostrare la sostanza ma allo stesso tempo anche di nasconderla in quanto essa non si esprime completamente, bensì vive solo grazie a un rapporto dinamico e attivo col suo opposto. Verso la realtà inizialmente non ci relazioniamo in modo astratto, ma come uomini storici, concreti e reali. L'insieme dei fatti con cui gli uomini si incontrano nel corso della loro esistenza reale viene definita «pseudoconcretezza», ovvero tutto ciò che risulta agli occhi di un'epoca e la caratterizza. La realtà, nel mondo della pseudoconcretezza, è un'unione tra fenomeno e sostanza, il primo considerato come illusione e la seconda come verità. Compito della filosofia e della scienza è far emergere l'essenza della sostanza che sta dietro i fenomeni, visto che se la sostanza si manifestasse direttamente la filosofia e la scienza sarebbero entrambe inutili. Su questo punto allora deve intervenire la dialettica come metodo orientato a separare e a scomporre i due aspetti della pseudoconcretezza e a capire la struttura della «cosa in sé», ossia la concretezza, come suo fine ultimo, così facendo la dialettica si presenta come un pensiero critico, contrario al dogmatismo e a ogni tipo di astrazione. Il mondo della pseudoconcretezza è il mondo dell'attività umana, fatta 
di prodotto e produzione, soggetto e oggetto, genesi e struttura; il mondo reale è il mondo della realizzazione della verità, della concretezza, che però non è data una volta per tutte, ma è in un continuo processo dove l'uomo inteso in senso storico produce la sua realizzazione. La distruzione della pseudoconcretezza è lo scopo non solo del marxismo, ma anche della fenomenologia e dell'esistenzialismo. Essa può avvenire in tre modi : 1) come umanizzazione dell'uomo grazie soprattutto alle rivoluzioni sociali 2) come pensiero dialettico, che distrugge il mondo feticizzato dell'apparenza 3) come realizzazione della verità nel singolo uomo. Dopo la distruzione della pseudoconcretezza grazie alla dialettica è possibile la liberazione dell'uomo dalle costrizioni feticistiche sulla realtà (KosíK, 1963 : 927).

\section{Conclusioni}

Nel clima particolare che si creò nella Praga degli anni '60 è stupefacente osservare come gli autori del CLP siano stati capaci, con mezzi relativamente ridotti, di sviluppare una serie di teorie convergenti verso concezioni sostanzialmente comuni, pur nelle rispettive differenze tra i membri sui temi e sugli approcci scelti. Questa caratteristica, già tipica per il periodo classico, permise al CLP di continuare a svolgere un ruolo significativo nella linguistica di quegli anni che gli dovrebbe essere riconosciuto anche oggi. Significativo sembra soprattutto il fatto che i dibattiti avvenuti in seno al CLP e i loro risultati preludano a quanto sarebbe diventato centrale verso la fine del decennio in questione nell'elaborazione di una teoria generale dei sistemi, valida e applicabile in più campi del sapere (BERTALANFFY, 1968 ; CHABROLLE-CERRETINI, 2010 : 17-18). Se non vi fossero state barriere esterne alla circolazione di queste idee, sia di ordine linguistico in alcuni casi che politico, lo sviluppo degli studi linguistici avrebbe probabilmente preso una direzione differente che forse vale la pena di recuperare nel contesto presente.

\section{BIBLIOGRAFIA}

BARKOVA Iryna (2008), Méthode phénomenologique dans la philosophie du langage du Cercle linguistique de Prague, Mémoire de maîtrise, Université du Québec à Montréal. Disponibile on line: http://www. archipel. uqam. ca/1622/1/M10642. pdf [visitato il 18. 3. 2013].

BĚLIČ Jaromír ; DOLEŽEL Lubomír ; PECIAR Štefan (eds.) (1962), Problémy marxistické jazykovědy [I problemi della linguistica marxista], Praha, Nakladatelství Československé akademie věd.

BERTALANFFY Ludwig von (1968), General System Theory. Foundations Development Applications, Harmondsworth, Penguin Books.

BlECHA Ivan (2003), Husserl a česká filosofie [Husserl e la filosofia ceca], Nakladatelství Olomouc.

ČERMÁK František (1995), Prague School of Linguistics Today, Linguistica Pragensia 1, p. 1-15.

ČERMÁK František (2003), Ferdinand de Saussure and the Prague Linguistic Structuralism, in : NeKUlA M. (ed.), Prager Strukturalismus. Prague Structuralism, Universitätsverlag Winter Heidelberg, p. 11-29. 
DANEŠ František (1966), The relation of centre and periphery as a language universal, in: VACHEK, Josef (ed.), Travaux linguistiques de Prague, Les problèmes du centre et de la périphérie du système de la langue, Academia, Éditions de l'Académie Tchécoslovaque des Sciences, Prague, p. 9-21.

DANEŠ František (1987), On Prague School Functionalism in Linguistics, in : DIRVEN René, FRIED Vilém (eds.), Functionalism in Linguistics, John Benjamins Publishing Company, Amsterdam/Philadelphia, p. 3-38.

HaJiČová Eva ; PANEVOVÁ Jarmila, SgAll Petr (2003), Úvod do teoretické a počítačové lingvistiky I. svazek - Teoretická lingvistika [Introduzione alla linguistica teorica e computazionale volume I - Linguistica teorica], Praha, Nakladatelství Klementinum.

HAVRÁNEK Bohuslav (1962), Aktuální metodologické problémy marxistické jazykovědy [Gli attuali problemi metodologici della linguistica marxista], in : BĚLIČ Jaromír, DOLEŽEL Lubomír, PECIAR Štefan (eds.), p. 9-19.

Chabrolle-CERRETINI Anne-Marie (2010), Les notions de centre et de périphérie : une certaine idée de l'organisation du système linguistique, in : RADIMSKÝ Jan (éd.), Centre et périphérie dans le système linguistique, Echo des études romanes, Vol. VI, No. 2, České Budějovice, Université de Bohême du Sud, p. 11-20.

JAKOBSON Roman (1966), Saggi di linguistica generale, Milano, Feltrinelli.

KARCEVSKIJ Sergej (1929), Du dualisme asymétrique du signe linguistique, in : Travaux du Cercle Linguistique de Prague I, p. 88-92 [rist. in VACHEK Josef (ed.) (1964), A Prague School Reader in Linguistics, Bloomington, Indiana Press University, p. 81-87].

KONRAD Kurt (1934), Svár obsahu a formy. Marxistické poznámky o novém formalismu [La saldatura tra contenuto e forma. Note marxiste su un nuovo formalismo], Středisko IV, n. 2, p. 56-65.

Kosík Karel (1963) [1965], Dialektika konkretního, Praha, Nakladatelství Československé akademie věd [trad. italiana Dialettica del concreto, Milano, Valentino Mompiani (a cura di Gianlorenzo Pacini)].

KosíK Karel (1962), Filosofické problémy struktury a systému [I problemi filosofici del concetto di struttura e di sistema], in : BĚLIČ Jaromír, DOLEŽEL Lubomír. PECIAR Štefan (eds.), p. 24-35.

MATHESIUS Vilém (1929), Funkční lingvistika [La linguistica funzionalistica], Sborník přednášek proslovených na Prvém sjezdu československých profesori̊ filosofie, filologie a historie v Praze 3. -7. dubna 1929, Praha, Stalý př́ípravný výbor sjezdový, p. 118-130.

MATHESIUS Vilém (1982), O potenciálnosti jevů jazykových [La potenzialità dei fenomeni linguistici], Jazyk, kultura a slovesnost, Praha, Odeon, p. 9-28.

MATHESIUS Vilém (1939), O tak zvaném aktuálním členěni větném [La cosiddetta Prospettiva funzionale della frase], Slovo a slovesnost 5, p. 171-174.

Novák Pavel, Sgall Petr (1968), On the Prague Functional Approach, in : DANEŠ, František et al. (eds.), Travaux linguistiques de Prague 3, Études structurales dédiées au VIe Congrès des slavistes, Prague, Academia Éditions de l'Académie Tchècoslovaque des Sciences, p. 291-297.

PATOČKA Jan (2009), Fenomenologický strukturalismus Romana Jakobsona [Lo strutturalismo fenomenologico di Roman Jakobson], in : PATOČKA Jan, 
Fenomenologické spisy II, Co je existence : publikované texty z let 1965-1977, Praha, Oikoymenh, p. 618-627.

RAYNAUD Savina (1990), Il Circolo linguistico di Praga, (1926-1939) : radici storiche e apporti teorici, Milano, Vita e Pensiero.

SAUSsure Ferdinand de, (2007), Corso di linguistica generale, Bari, Editori Laterza.

SKALIČKA Vladimír (1948), Kodaňský strukturalismus a Pražská škola [Lo strutturalismo di Copenhagen e la Scuola di Praga], Slovo a slovesnost 10, p. 135-142.

SORNICOLA Rosanna, SvobODA Aleš (1991), Il campo di tensione : la sintassi della Scuola di Praga, Napoli, Liguori.

ŠOLTYS Otakar (1991), Kulturní kontext Pražského lingvistického kroužku [Il contesto culturale del Circolo Linguistico di Praga], Slovo a slovesnost 52, p. 198-201.

VACHEK Josef (1966), The linguistic school of Prague, Bloomington and London, Indiana University Press.

VACHEK Josef (1999), Prolegomena $k$ dějinám Pražské školy jazykovědné [Prolegomeni per una storia della Scuola di Praga], Jinočany, Nakladatelství $\mathrm{H} \& \mathrm{H}$.

VAN Der Berghe Pierre L. (1963), Dialectic and functionalism : toward a theorical synthesis, American Sociological Review, vol. 28, n. 5, p. 695-705. 